\title{
Comportement non-linéaire d'un modèle de Boussinesq étendu
}

\author{
Gomi P. ${ }^{(a)}$, Sergent P. ${ }^{(a)}$, Meftah K. ${ }^{(b)}$ \\ (a) Centre d'Etude Technique Maritimes Et Fluviales, \\ 2 Bd Gambetta, B.P. 60039, 60321 Compiègne Cedex, \\ Tél: 03.44.92.60.30 E-mail:philippe.sergent@equipement.gouv.fr \\ (b) Université de Technologie de Compiègne, Laboratoire d'Hydraulogie Numérique, \\ 66 Av. de Landshut, 60200 Compiègne
}

\section{Résumé}

Un nouveau type de modèle de Boussinesq a été développé en décomposant les composantes horizontales de la vitesse sur une série de fonctions ne dépendant que de $\mathrm{z}$. Cet artifice mathématique permet d'améliorer son comportement dispersif en étendant son domaine de validité fréquentiel et également d'améliorer la reproduction des profils verticaux de vitesses et son aptitude à faire face au shoaling linéaire et aux fortes pentes. L'objet de cet article est d'abord de présenter les bases théoriques de ce modèle aux éléments finis mais également d'étudier son comportement non-linéaire d'ordre 2.

\begin{abstract}
$\underline{\text { Abstract }}$
A new type of extended Boussinesq model has been developed using a mathematical artifice that decomposes each vertical component of velocity on a series of functions on the water depth. This mathematical artifice enables to improve its linear dispersion behaviour by increasing its frequencial range of validity and also to improve its ability to reproduce vertical velocity profile and to deal with linear shoaling and steep slopes. The aim of this paper is to present the theoretical bases of this finite elements model and also to study its non-linear $2 \backslash$ textsuperscript $\{$ nd $\}$ order behaviour.
\end{abstract}

\section{Introduction}

Depuis quelques années, beaucoup d'efforts portent sur l'amélioration des modèles de Boussinesq afin d'étendre leur domaine fréquentiel d'application à des ondes en eaux plus profondes ce qui revient la plupart du temps à améliorer leur comportement dispersif. La manière la plus commune d'étendre un modèle de Boussinesq consiste à ajouter aux équations initiales des termes supplémentaires qui dérivent la plupart du temps de développements de Padé(Witting 1984, Murray 1989, Madsen et al. 1991, Nwogu 1993, Sch $\backslash H\{a\} f f e r$ and Madsen 1995, Agnon et al. 1999, Gobbi and Kirby 2000, Madsen et al. 2002.).

Nous avons développé un nouveau type de modèle étendu de Boussinesq dont le fondement consiste à décomposer les composantes de la vitesse et la pression nonhydrostatique sur une série de fonctions dépendant uniquement de $z$, ce qui permet de remplacer la dimension verticale par un noeud unique enrichi en degrés de liberté. Cet artifice mathématique permet de d'améliorer implicitement la relation de dispersion linéaire du modèle qui tend vers l'équation de dispersion théorique lorsqu'on augmente le nombre $\mathrm{N}$ de fonctions constituant la série.

Une étude dispersive linéaire du modèle montre également que, pour chaque série de fonctions utilisée, il existe une fréquence de coupure ce qui signifie que les composantes du signal dont la fréquence est supérieure à cette fréquence critique sont amorties par le modèle. Dans le cas d'un modèle «classique» de type Boussinesq étendu, la différence entre la relation de dispersion du modèle et la relation théorique augmente lorsque la fréquence augmente ce 
qui peux mener à des résultats aléatoires. Il s'agit de deux approches opposées, l'une ne prenant pas en compte certaines composantes hautes-fréquences, l'autre les prenant en compte mais de façon inappropriée.

Nous avons également étudié le comportement de notre modèle étendu de Boussinesq non seulement en ce qui concerne la

modélisation du shoaling linéaire mais également sur sa capacité à bien traiter les effets de pentes lorsque celles-ci deviennent importantes. De la $\mathrm{m} \backslash^{\wedge}$ eme façon que pour l'étude de la relation de dispersion linéaire nous avons constaté que le comportement de notre modèle face au shoaling tend à se rapprocher implicitement de la théorie linéaire lorsqu'on augmente le nombre $\mathrm{N}$ de fonctions et cela sans rajouter ni calibrer de termes supplémentaires dans les équations initiales. Le modèle a ensuite été utilisé pour reproduire le cas-test de Booij (1983) afin d'étudier sa pertinence face à des pentes élevées. Nous avons trouvé des résultats très proches de ceux donnés par le modèle numérique 3D développé par Booij et donc une bonne reproduction des effets de pente non-linéaires.

Afin de valider notre modèle sur un cas-test de propagation de la houle assez difficile, nous l'avons utilisé sur le cas-test de Dingemans (1994) reproduisant la déformation d'un train de houle régulière suite à son passage sur une barre trapézoidale. Nous observons naturellement une amélioration des résultats lorsque nous augmentons le nombre de fonctions et nous obtenons de très bons résultats pour les tests $\mathrm{A}$ et $\mathrm{B}$ lorsque nous prenons 3 fonctions sur la série.(voir figure 1).

Avec une seule fonction nous retrouvons le modèle de Boussinesq de Peregrine (1967).
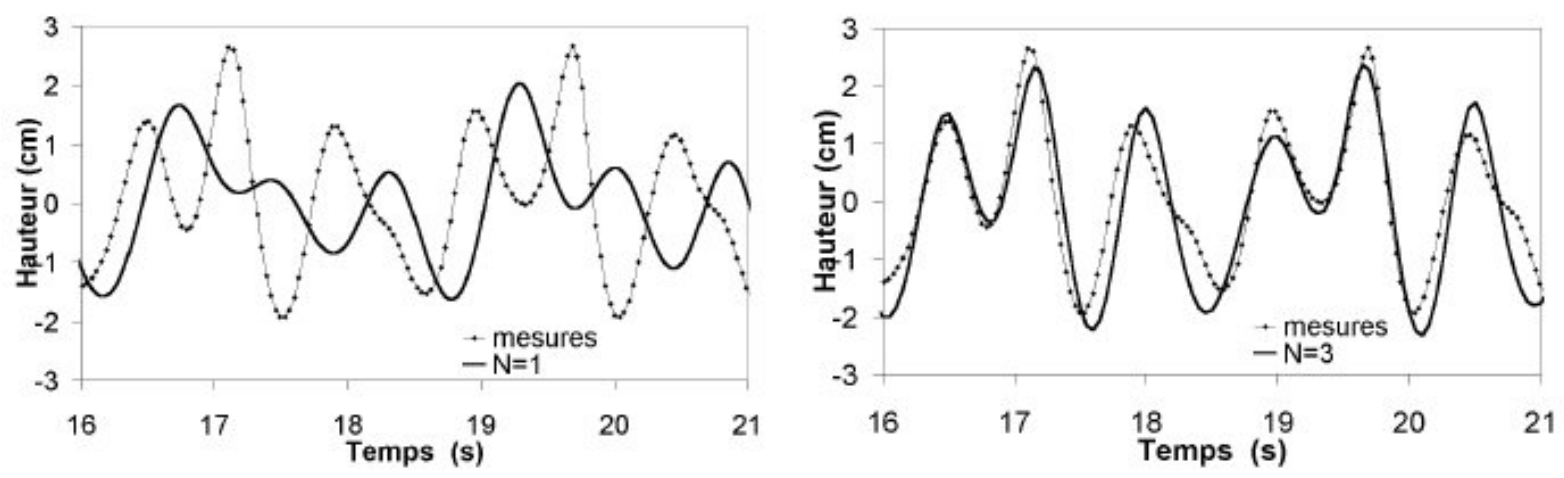

Figure 1: Test $B, x=17.3 m$, résultats pour $N=1$ et $N=3$

Un autre aspect intéressant de ce modèle étendu est que le choix des fonctions constituant les séries n'est pas limité et que les utilisateurs de ce modèle peuvent introduire de nouvelles séries de fonctions et faire varier le nombre de ces fonctions. Ce modèle est également capable de reproduire plus fidèlement les variations des paramètres physiques suivant $\mathrm{z}$ car l'utilisateur peut directement choisir les fonctions qui correspondent le mieux à la nature physique de l'écoulement avec par exemple des fonctions logarithmiques pour un écoulement fluvial ou des fonctions hyperboliques pour une étude de houle ou d'ondes évanescentes. Parmi les différents choix de base, nous privilégions les Polynômes de Legendre qui fournissent le meilleur compromis: précision, simplicité et temps de calcul. Avec une base de fonctions hyperboliques, nous retrouvons les modèles de Nadaoka (1994) et Massel (1993).

L'objet de cet article est tout d'abord de présenter les fondements théoriques de ce modèle étendu de Boussinesq sans écrire tous les termes qui apparaissent au cours du développement et qui jouent un rô le dans le modèle numérique. Cet article propose également de compléter 
les analyses linéaires déjà réalisées (dispersion, profil vertical de vitesse, shoaling) par une analyse d'ordre $2 \mathrm{du}$ comportement non-linéaire de ce modèle sur fond plat.

\section{Système d'équations à résoudre}

Pour simplifier et raccourcir l'écriture des équations nous les exprimons ici dans un référentiel bidimensionnel vertical. Dans le référentiel Oxz, les équations de conservation de la masse et de conservation de la quantité de mouvement peuvent s'écrire:

$$
\left\{\begin{array}{l}
\frac{\partial u}{\partial t}+u \frac{\partial u}{\partial x}+w \frac{\partial u}{\partial z}+\frac{1}{\rho} \frac{\partial p}{\partial x}=0 \\
\frac{\partial w}{\partial t}+u \frac{\partial w}{\partial x}+w \frac{\partial w}{\partial z}+\frac{1}{\rho} \frac{\partial p}{\partial z}-g=0 \\
\frac{\partial u}{\partial x}+\frac{\partial w}{\partial z}
\end{array}\right.
$$

où $\mathrm{p}, \mathrm{u}, \mathrm{w}, \mathrm{g}$ et $\mathrm{p}$ sont respectivement la pression, les composantes horizontales et verticales de la vitesse, la constante de pesanteur et la masse volumique du fluide.

La pression à l'intérieur du fluide peut se décomposer de la façon suivante:

$$
p=\rho g(h-z)+p_{a t m}+\rho p^{\prime}
$$

avec $\mathrm{p}_{\mathrm{atm}}$ la pression atmosphérique à la surface libre et pp' la composante non-hydrostatique

L'équation de continuité cinématique à la surface libre peut s'exprimer de la façon suivante:

$$
\frac{\partial h}{\partial t}+u_{s} \frac{\partial h}{\partial x}-w_{s}=0
$$

où $\mathrm{h}(\mathrm{x}, \mathrm{t})$ est le niveau de la surface libre et $\left(\mathrm{u}_{\mathrm{s}}, \mathrm{W}_{\mathrm{s}}\right)$ sont les composantes de la vitesse à la surface libre.

La condition de continuité de la pression à la surface libre se traduit par:

$$
p(x, h)=p_{\text {atm }} \quad \text { et donc } \quad p^{\prime}(x, h)=0
$$

L'équation de continuité cinématique au fond s'exprime de la façon suivante:

$$
u_{b} \frac{\mathrm{d} z_{b}}{\mathrm{~d} x}-w_{b}=0
$$

avec $\mathrm{z}_{\mathrm{b}}(\mathrm{x})$ le niveau du fond supposé constant dans le temps et $\left(\mathrm{u}_{\mathrm{b}}, \mathrm{w}_{\mathrm{b}}\right)$ les composantes de la vitesse au fond.

\section{La méthode h-s}

Le noyau de notre modèle repose sur la décomposition des composantes horizontales de la vitesse sur des séries de fonctions ne dépendant que de $\mathrm{z}$ :

$$
u\left(x, t, z, z_{b}, h\right)=\phi_{i}\left(z, z_{b}, h\right) u_{i}(x, t)
$$


où la notation $\mathrm{A}_{\mathrm{i}} \mathrm{B}_{\mathrm{i}}$ est une notation raccourcie de $\sum_{i=1}^{N} A i B i$ avec $\mathrm{N}$ l'ordre de discrétisation sur la verticale.

$\emptyset_{\mathrm{i}}(\mathrm{z}, \mathrm{z}$ b, $\mathrm{h})$ est une série de fonctions qui permet de mieux restituer le comportement de l'écoulement sur la verticale car nous pouvons directement choisir des fonctions qui correspondent le mieux à la nature physique de l'écoulement à savoir des fonctions logarithmiques pour un écoulement fluvial ou des fonctions hyperboliques pour une étude de houle ou d'ondes évanescentes ou par défaut des polynômes de Legendre.

\subsection{Equation de conservation de lamasse}

Si nous intégrons l'équation de la masse du système (1) du fond à la surface libre en utilisant l'équation (3), nous obtenons l'équation:

$$
\frac{\partial h}{\partial t}+\frac{\partial q}{\partial x}=0 \quad \text { avec } \quad q=\int_{z_{b}}^{h} u \mathrm{~d} z
$$

\subsection{Composante verticale de la vitesse}

L'intégration de la troisième équation du système (1) du fond $\mathrm{z}_{\mathrm{b}}$ à la côte $\mathrm{z}$ permet d'exprimer la composante verticale de la vitesse en fonction de différentes variables dépendant uniquement de $\mathrm{z}, \mathrm{z}_{\mathrm{b}}$ et $\mathrm{h}$.

$$
\begin{aligned}
\int_{z_{b}}^{z}\left(\frac{\partial u}{\partial x}+\frac{\partial w}{\partial z}\right) \mathrm{d} z=0 \quad \text { soit } & w-w_{b}=\psi_{i} w_{i}-u_{i} \frac{\partial z_{b}}{\partial x} \int_{z_{b}}^{z} \frac{\partial \phi_{i}}{\partial z_{b}} \mathrm{~d} z+p_{i} u_{i} \frac{\partial h}{\partial x} \\
\text { où } \quad \psi_{i}\left(z, h, z_{b}\right)=\int_{z_{b}}^{z} \phi_{i} \mathrm{~d} z, & w_{i}=-\frac{\partial u_{i}}{\partial x}, \quad p_{i}\left(z, h, z_{b}\right)=-\int_{z_{b}}^{z} \frac{\partial \phi_{i}}{\partial h} \mathrm{~d} z \\
\text { d'après l'equation (5) nous savons que } & w_{b}=u_{b} \frac{\mathrm{d} z_{b}}{\mathrm{~d} x}=\phi_{i}^{b} u_{i} \frac{\mathrm{d} z_{b}}{\mathrm{~d} x} \text { ce qui signifie que : }
\end{aligned}
$$

$$
\begin{gathered}
w=\psi_{i} w_{i}+q_{i} u_{i} \frac{\partial z_{b}}{\partial x}+p_{i} u_{i} \frac{\partial h}{\partial x} \\
\text { avec } \quad q_{i}\left(z, h, z_{b}\right)=\phi_{i}^{b}-\int_{z_{b}}^{z} \frac{\partial \phi_{i}}{\partial z_{b}} \mathrm{~d} z \quad \text { et } \phi_{i}^{b}=\phi_{i}\left(z_{b}, h, z_{b}\right)
\end{gathered}
$$

\subsection{Expression de la composante non-hydrostatique de la pression}

La $2^{\text {ème }}$ équation de la conservation de la quantité de mouvement du système (1) peut s'écrire:

$$
\frac{\partial w}{\partial t}+\frac{\partial u w}{\partial x}+\frac{\partial w^{2}}{\partial z}+\frac{\partial p^{\prime}}{\partial z}=0
$$

Il suffit une fois encore d'intégrer cette équation de $\mathrm{z}$ à la surface libre et d'utiliser l'équation (9) pour obtenir:

$$
\begin{aligned}
-p^{\prime}=A_{i} u_{i} & +B_{i} w_{i}+C_{i} \frac{\partial u_{i}}{\partial t}+D_{i} \frac{\partial w_{i}}{\partial t}+E_{i j} u_{i} u_{j}+F_{i j} u_{i} w_{j}+G_{i j} \frac{\partial u_{i} u_{j}}{\partial x} \\
& +H_{i j} \frac{\partial u_{i} w_{j}}{\partial x}+K_{i j} u_{i} u_{j}+L_{i j} w_{i} w_{j}+M_{i j} u_{i} w_{j}
\end{aligned}
$$

où les fonctions $A_{i}, B_{i}, C_{i}, D_{i}, E_{i j}, F_{i j}, G_{i j}, H_{i j}, K_{i j}, L_{i j}$ et $M_{i j}$ sont des fonctions ne dépendant que de $z$, h et $z_{b}$ et pouvant s'exprimer en fonctions des variables $\emptyset_{i}, \psi_{i} p_{i}$ et $q_{i}$ 


\subsection{Système à résoudre}

Le problème étudié revient à résoudre le système suivant:

$$
\left\{\begin{aligned}
\frac{\partial u}{\partial t}+\frac{\partial u^{2}}{\partial x}+\frac{\partial u w}{\partial z}+\frac{1}{\rho} \frac{\partial p}{\partial x} & =0 \\
\frac{\partial h}{\partial t}+\frac{\partial q}{\partial x} & =0
\end{aligned}\right.
$$

avec $\mathrm{p}=\rho \mathrm{g}(\mathrm{h}-\mathrm{z})+\mathrm{p}_{\mathrm{atm}}+\rho \mathrm{p}$ ' et où les variables $\mathrm{u}, \mathrm{w}$ et $\mathrm{p}^{\prime}$ vérifient respectivement les équations (6), (9) et (11).

\section{$\underline{4 . A n a l y s e ~ n o n-l i n e ́ a i r e ~ d u ~ s e c o n d ~ o r d r e ~}$}

\subsection{Hypothèse et simplifications des équations}

Nous écrivons le niveau d'eau $\mathrm{h}$ et les $\mathrm{N}$ composantes horizontales de la vitesse $\mathrm{u}_{\mathrm{i}}$ en écrivant $\theta=\omega t-\kappa x$ et en considérant que $\epsilon<<1$ :

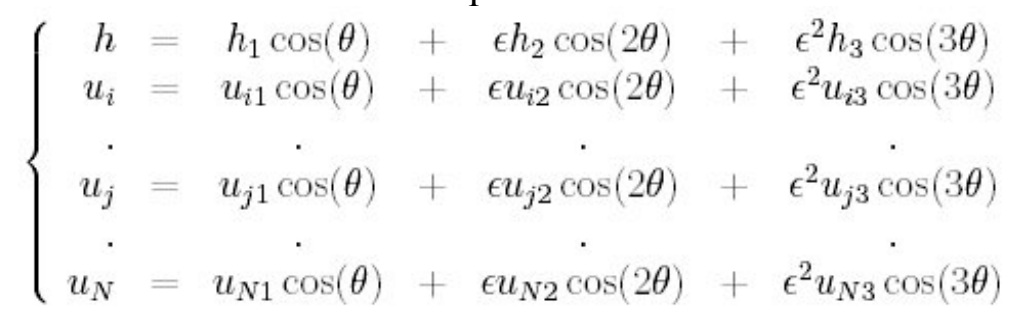

On considère que l'écoulement se fait sur un fond plat n'évoluant pas dans le temps ce qui implique que $\frac{\partial z b}{\partial x}=0$ et $\frac{\partial z b}{\partial t}=0$.On élimine également du système (12) tous les termes d'ordre plus grand que 2 qui n'interviendront pas dans cette analyse non-linéaire d'ordre 2 c'est à dire tous les termes produits d'au moins 3 fonctions inconnues (où de leurs dérivées).Il reste alors le système suivant lorsqu'on effectue le remplacement $w_{i}=-\frac{\partial u_{i}}{\partial x}$ :

$$
\left\{\begin{array}{c}
\phi_{i} \frac{\partial u_{i}}{\partial t}+\phi_{i} \phi_{j} u_{i} \frac{\partial u_{j}}{\partial x}-\psi_{i} \frac{\partial \phi_{j}}{\partial z} \frac{\partial u_{i}}{\partial x} u_{j}+g \frac{\partial h}{\partial x}+A_{i}^{5} \frac{\partial h}{\partial x} \frac{\partial^{2} u_{i}}{\partial t \partial x}+A_{i}^{5} \frac{\partial^{2} h}{\partial x^{2}} \frac{\partial u_{i}}{\partial t}-\frac{\partial D_{i}}{\partial h} \frac{\partial h}{\partial x} \frac{\partial^{2} u_{i}}{\partial t \partial x} \\
-D_{i} \frac{\partial^{3} u_{i}}{\partial t \partial x^{2}}-H_{i j}\left(\frac{\partial^{2} u_{i}}{\partial x^{2}} \frac{\partial u_{j}}{\partial x}+u_{i} \frac{\partial^{3} u_{j}}{\partial x^{3}}+2 \frac{\partial u_{i}}{\partial x} \frac{\partial^{2} u_{j}}{\partial x^{2}}\right)+L_{i j}\left(\frac{\partial u_{i}}{\partial x} \frac{\partial^{2} u_{j}}{\partial x^{2}}+\frac{\partial^{2} u_{i}}{\partial x^{2}} \frac{\partial u_{j}}{\partial x}\right)=0 \\
\frac{\partial h}{\partial t}+\left(h-z_{b}\right) \frac{\partial u_{1}}{\partial x}+\frac{\partial h}{\partial x} u_{i} \phi_{i}^{h}=0 \\
\text { avec } \phi_{i}^{h}=\phi_{i}\left(h, h, z_{b}\right) \quad D_{i}\left(z, h, z_{b}\right)=\int_{z}^{h} \psi_{i} \mathrm{~d} z \quad A_{i}^{5}\left(z, h, z_{b}\right)=\int_{z}^{h} p_{i} \mathrm{~d} z \\
H_{i j}\left(z, h, z_{b}\right)=\int_{z}^{h} \phi_{i} \psi_{j} \mathrm{~d} z \quad \psi_{i}^{h}=\psi_{i}\left(h, h, z_{b}\right) \quad L_{i j}\left(z, h, z_{b}\right)=\psi_{i}^{h} \psi_{j}^{h}-\psi_{i} \psi_{j}
\end{array}\right.
$$

\subsection{Adimentionnement et multiplication des équations}

On rappelle que la hauteur d'eau totale vaut $\mathrm{H}=\mathrm{h}-\mathrm{Z}_{\mathrm{b}}$ ou $\mathrm{H}=\mathrm{h}+\mathrm{d}$ avec $\mathrm{d}=-\mathrm{Z}_{\mathrm{b}}$.

Pour les variables $\mathrm{x}, \mathrm{z}, \mathrm{t}, \mathrm{d}, \mathrm{h}$, et $\mathrm{u}$ nous effectuons respectivement les changements de variables suivants:

$$
\frac{x}{l_{0}}, \quad \frac{z}{d_{0}}, \quad \frac{\sqrt{g d_{0}}}{l_{0}} t, \quad \frac{d}{d_{0}}, \quad \frac{h}{a_{0}} \quad \text { et } \quad \frac{d_{0}}{a_{0}{\sqrt{g d_{0}}}} u
$$

où les nombres $\mathrm{d}_{0}, 1_{0}$ et $\mathrm{a}_{0}$ sont respectivement les profondeurs d'eau, longueur d'onde et amplitude de la houle caractéristiques. 

s'écrire:

On fait également intervenir les deux paramètres adimensionnels $\epsilon$ et $\mu$ qui peuvent

$$
\epsilon=\frac{a_{0}}{d_{0}}, \mu=\frac{d_{0}}{l_{0}} \text { et donc } \epsilon \mu=\frac{a_{0}}{l_{0}}
$$

On peut également facilement prouver que Hs'écrit $\mathrm{d}_{0}(\epsilon \mathrm{h}+\mathrm{d})$ après changement de variables.

Pour se ramener de l'intervalle $\left[\mathrm{z}_{\mathrm{b}} ; \mathrm{h}\right]$ à l'intervalle $[-1 ; 1]$, nous effectuons le changement de variable $z=\frac{H}{2}(\xi+1)+z b$ qui est particulièrement intéressant pour les polynômes de Legendre qui sont définis entre -1 et 1 .

Ainsi par exemple la fonction $\psi_{\mathrm{i}}\left(\mathrm{z}, \mathrm{h}, \mathrm{z}_{\mathrm{b}}\right)$ peut également s'écrire:

$$
\psi_{i}\left(z, h, z_{b}\right)=\int_{z_{b}}^{z} \phi_{i} \mathrm{~d} z=\frac{H}{2} \int_{-1}^{\xi} \Phi_{i} \mathrm{~d} \xi=\frac{H}{2} \Psi_{i}(\xi)
$$

et la fonction $\mathrm{H}_{\mathrm{ij}}\left(\mathrm{z}, \mathrm{h}, \mathrm{Z}_{\mathrm{b}}\right)$ peut de la même façon s'écrire:

$$
H_{i j}\left(z, h, z_{b}\right)=\int_{z}^{h} \phi_{i} \psi_{j} \mathrm{~d} z=\frac{H^{2}}{4} \int_{\xi}^{1} \Phi_{i} \Psi_{j} \mathrm{~d} \xi=\frac{H^{2}}{4} \mathrm{H}_{i j}(\xi)
$$

Pour obtenir autant d'équations que d'inconnues on applique l'opérateur $\int_{z b}^{h} \phi k(z)(*) d z$ à la première équation du système (14) précédent pour obtenir finallement:

$$
\left\{\begin{aligned}
& \mathbf{F}_{i k} \frac{\partial u_{i}}{\partial t}+\mathbf{F}_{i j k} \epsilon u_{i} \frac{\partial u_{j}}{\partial x}+\mathbf{Q}_{i j k} \epsilon \frac{\partial u_{i}}{\partial x} u_{j}+\Psi_{k}^{1} \frac{\partial h}{\partial x}+\frac{1}{4} \mathbf{A}_{i k}^{5} \epsilon \mu^{2}(\epsilon h+d)\left(\frac{\partial h}{\partial x} \frac{\partial^{2} u_{i}}{\partial t \partial x}+\frac{\partial^{2} h}{\partial x^{2}} \frac{\partial u_{i}}{\partial t}\right) \\
&+\frac{1}{4} \mathbf{D}_{i k} \mu^{2}(\epsilon h+d)^{2} \frac{\partial^{3} u_{i}}{\partial t \partial x^{2}}+\frac{1}{4} \mathbf{D}_{i k}^{\prime} \epsilon \mu^{2}(\epsilon h+d) \frac{\partial h}{\partial x} \frac{\partial^{2} u_{i}}{\partial t \partial x}+\frac{1}{2} \mathbf{D}_{i k} \epsilon \mu^{2}(\epsilon h+d) \frac{\partial h}{\partial x} \frac{\partial^{2} u_{i}}{\partial t \partial x} \\
&+\frac{1}{4} \mathbf{H}_{i j k} \epsilon \mu^{2}(\epsilon h+d)^{2}\left(\frac{\partial^{2} u_{i}}{\partial x^{2}} \frac{\partial u_{j}}{\partial x}+u_{i} \frac{\partial^{3} u_{j}}{\partial x^{3}}+2 \frac{\partial u_{i}}{\partial x} \frac{\partial^{2} u_{j}}{\partial x^{2}}\right) \\
&+\frac{1}{4} \mathbf{L}_{i j k} \epsilon \mu^{2}(\epsilon h+d)^{2}\left(\frac{\partial u_{i}}{\partial x} \frac{\partial^{2} u_{j}}{\partial x^{2}}+\frac{\partial^{2} u_{i}}{\partial x^{2}} \frac{\partial u_{j}}{\partial x}\right)=0 \\
& \frac{\partial h}{\partial t}+(\epsilon h+d) \frac{\partial u_{1}}{\partial x}+\epsilon u_{i} \Phi_{i}^{1} \frac{\partial h}{\partial x}=0
\end{aligned}\right.
$$

\subsection{Application à l'analyse non-linéaire d'ordre 2}

On injecte le système (13) dans le système (16). Si on ne conserve que les termes d'ordre 1 (sans $\epsilon$ )et que l'on résout le système alors obtenu, cela permet d'obtenir la relation de dispersion linéaire du modèle (déterminant de la matrice trouvée devant être nul) ainsi que les vecteurs propres $f i$ permettant de reconstituer le profil vertical de la vitesse.Le cas qui nous intéresse ici consiste à ne conserver que les termes d'ordre 2 en \$lepsilon\$ et de résoudre le système suivant:

$$
\left[\begin{array}{cc}
2 \omega & -2 k d \\
-4 k \Psi_{k}^{1} & 2 \omega\left(\mathbf{F}_{i k}-4 \kappa^{2} \mathbf{D}_{i k}\right)
\end{array}\right]\left[\begin{array}{c}
h_{2} \\
u_{i 2}
\end{array}\right]=\left[\begin{array}{c}
F_{1} \\
F_{2}^{k}
\end{array}\right]
$$


Sachant que $u_{1}^{i}=f i \frac{\omega}{k d} h 1$ et que l'on pose $k=k \mu d$, on peut alors écrire $F 1$ et $F_{2}^{k}$ sous la forme:

$$
\left\{\begin{array}{l}
F_{1}=\frac{\omega h_{1}^{2}}{2 d}\left(f_{1}+f_{i} \Phi_{i}^{1}\right) \\
F_{2}^{k}=\frac{\omega^{2} h_{1}^{2}}{2 k d^{2}}\left[f_{i} f_{j}\left(\mathbf{F}_{i j k}+\mathbf{Q}_{i j k}\right)+\kappa^{2} f_{i}\left(\mathbf{D}_{i k}+\frac{\mathbf{A}_{i k}^{5}}{2}+\frac{\mathbf{D}_{i k}^{\prime}}{4}\right)-\kappa^{2} f_{i} f_{j}\left(\mathrm{H}_{i j k}+\frac{\mathbf{L}_{i j k}}{2}\right)\right]
\end{array}\right.
$$

Il suffit ensuite d'inverser le système linéaire (17) à $\mathrm{N}+1$ inconnues pour $\mathrm{N}+1$ équations ce qui permet alors d'exprimer $h_{2}$ et les $\mathrm{N} \mathrm{u}_{\mathrm{i} 2}$ en fonctions des paramètres du problème.

On peut ensuite comparer le comportement $d u h_{2}$ de notre modèle avec la formule de la théorie de Stokes du second ordre qui peut s'écrire de la façon suivante:

$$
h_{2}^{\text {Stokes }}=\frac{1}{4} \frac{h_{1}^{2}}{d} \kappa \operatorname{coth}(\kappa)\left(3 \operatorname{coth}^{2}(\kappa)-1\right)
$$

La Figure 2 montre le quotient $\mathrm{h}_{2} / \mathrm{h}_{2}{ }^{\text {Stokes }}$ pour un nombre de fonctions utilisées $\mathrm{N}$ variant de 1 à 5 .

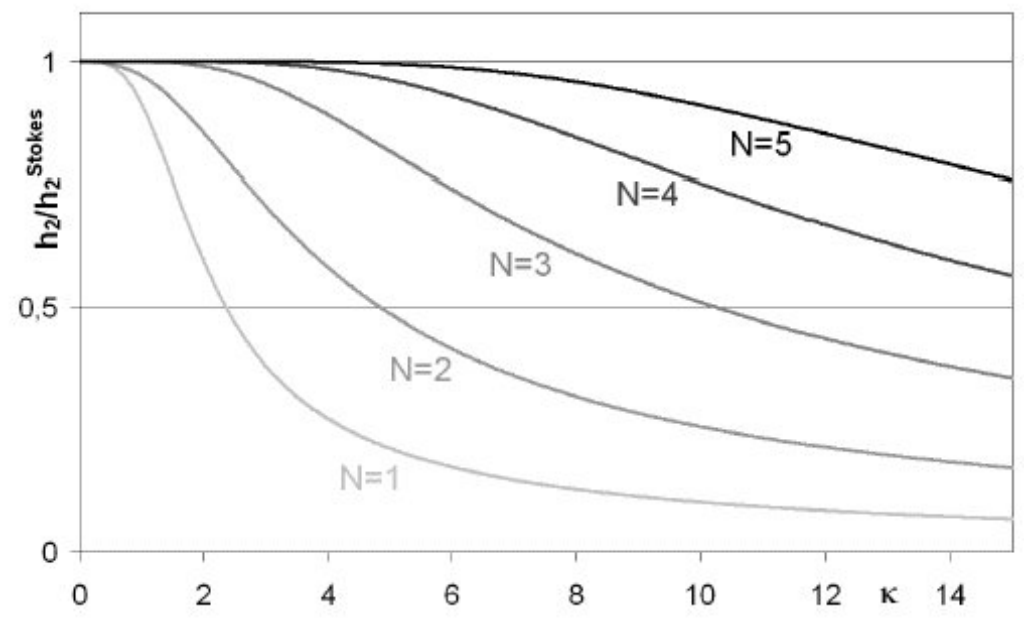

Figure 2: Rapport $h_{2} / h_{2}^{\text {Stokes }}$ pour $N=1$ à $N=5$

Nous constatons avec ces courbes que plus le nombre $\mathrm{N}$ de fonctions augmente plus le comportement non-linéaire de notre modèle s'améliore et tend vers la formule théorique donnée par la théorie de Stokes. Ainsi pour $\mathrm{N}=5$ on peut admettre que notre modèle a un très bon comportement non-linéaire jusqu'à $\mathrm{k}=10$ et son comportement s'améliorera encore si on augmente N. Beaucoup de modèles étendus de Boussinesq actuels (voir par exemple Madsen et Schaffer ${ }^{15}$ ) n'arrivent pas à obtenir un tel domaine de validité pour la non-linéarité de second ordre et cela malgré de nombreuses calibrations et ajouts de termes. L'amélioration du comportement non-linéaire de notre modèle en fonction du nombre de fonctions se fait implicitement et ne requiert aucune calibration ni ajouts de termes dans les équations de base. Ainsi, par exemple, les figures 3 et 4 montrent le même genre d'amélioration respectivement pour la dispersion linéaire et pour le shoaling linéaire (Meftah et al. ${ }^{9} 2004$ ). Pour mieux comprendre les paramètres physiques concernés par ces figures, il convient de rappeler leur définition: 
C est la célérité de l'onde, $C_{0}=\sqrt{g H}, \gamma_{0}(k)=-\frac{H}{A} \frac{d A}{d x} \frac{d x}{d z b}$ est le gradient de shoaling linéaire définit par Madsen et Sorensen ${ }^{16}$ (1992) avec A l'amplitude de l'onde considérée progressive, $a=\frac{\omega_{2 H}}{g}$ est un paramètre adimensionnel.

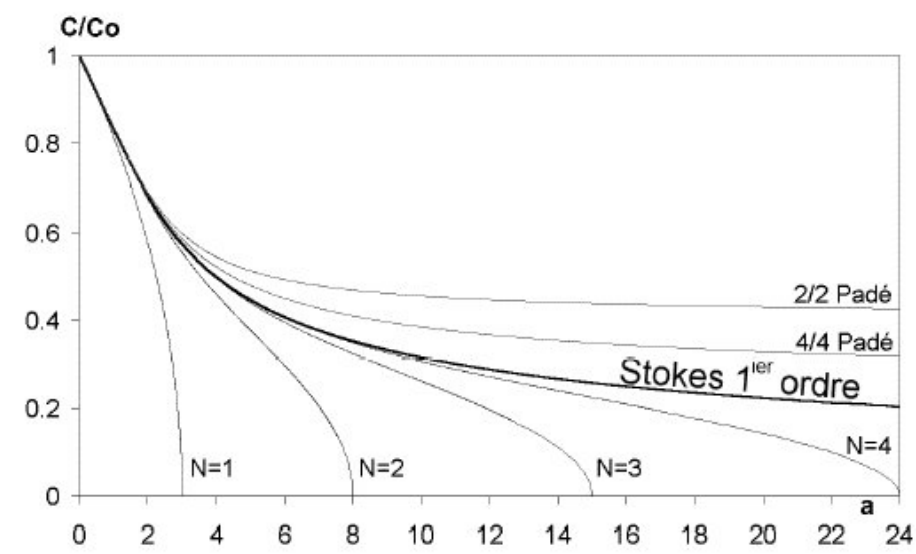

Figure 3: Représentation de la relation de dispertion pour $N=1$ et $N=5$

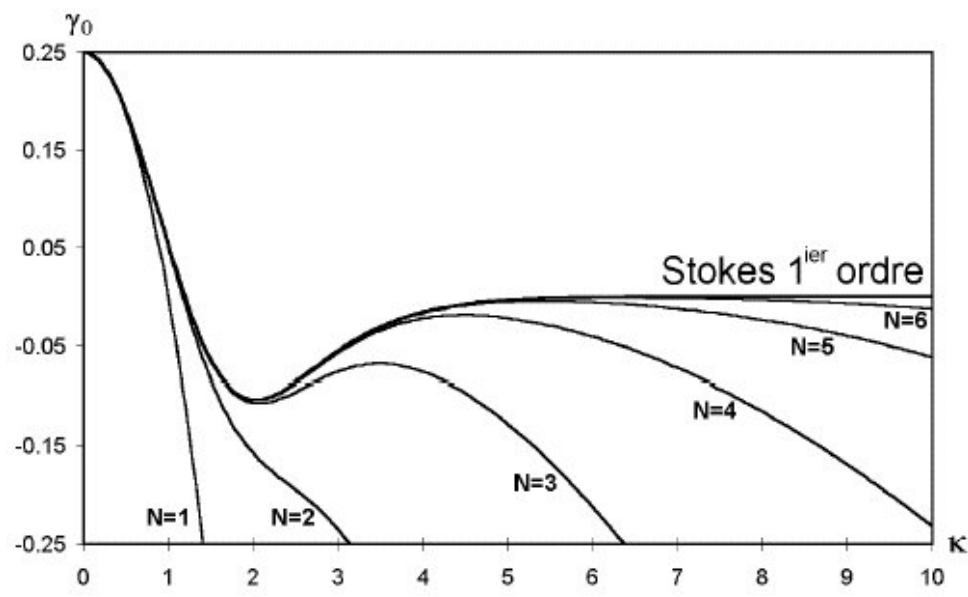

Figure 4: Coefficient de shoaling linéaire $\gamma_{0}$ pou $N=1$ et $N=5$

\section{Conclusion}

Le travail présenté dans cet article porte essentiellement sur une analyse non-linéaire d'ordre 2 d'un nouveau type de

modèles de Boussinesq étendu précédée par une présentation succincte de ses fondements théoriques. Pour ce modèle les composantes horizontales de la vitesse sont décomposées sur des séries de fonctions ne dépendant que de $\mathrm{z}$ ce qui permet de remplacer chaque verticale par un noeud unique enrichi en degrés de liberté.Le premier avantage de ce type de modèle est qu'un modèle $2 \mathrm{D}$ horizontal suffit pour étudier un problème $3 \mathrm{D}$ ce qui facilite le prétraitement et donne lieu à des calculs moins longs. Le deuxième avantage est que qu'il génère implicitement une relation de dispersion améliorée qui tend vers l'équation linéaire de Stokes quand le nombre de fonctions $\mathrm{N}$ devient suffisamment grand. De la même façon, on observe une amélioration implicite du comportement de son shoaling linéaire, de sa capacité à traiter les effets non-linéaires de pentes de fond plus abruptes et à représenter les profils verticaux de vitesses. Un autre aspect intéressant de ce modèle consiste aux choix de fonctions disponibles et non figées suivant la nature physique des phénomènes à étudier. 
L'analyse non-linéaire du second ordre qui est décrite dans ce travail montre une amélioration implicite du comportement non-linéaire de ce modèle en fonction du nombre $\mathrm{N}$. Une étude d'ordre 3 suivra et devrait donner lieu aux mêmes typesde conclusions.

\section{Références}

1.Witting, J.M., 1984, A unified model for the evolution of nonlinear water waves. Journal of Computational Physics, 50, 203-236.

2.Murray, R.J., 1989. Short wave modelling using new equations of Boussinesq type. $9^{\text {th }}$ Australian Conference on Coastal and Ocean Engineering, Institution of Engineering, Adelaïde, Australia 331-336.

3.Madsen, P.A., Murray,R., Sorensen, O.R.,1991. A new form of the Boussinesq equations with improved linear dispersion characteristics. Coastal Engineering, vol 15, 371388.

4.Nwogu, O., 1993. Alternative form of boussinesq equations for nearshore wave propagations. Journal of Waterway, port, Coastal and Ocean Engineering 119, 618-638.

5.Agnon Y., Madsen P.A., Schäffer H.A.,\}]1999. A new approach to high-order Boussinesq models. Journal of Fluid Mechanics, Vol 399, 319-333.

6.Gobbi, M.F., Kirby, J.T., Wei, G.,\}]2000. A fully nonlinear Boussinesq model for surface waves Part2. Journal of Fluid Mechanics, Vol 405, 181-210.

7.Madsen, P.A., Bingham, H.B., Liu, H.,2002. A new Boussinesq method for fully nonlinear waves from shallow to deep water. Journal of Fluid Mechanics, Vol 462, 1-30.

8.Meftah, K.,1998. Modélisation tridimensionnelle de l'hydrodynamique et du transport par suspension. PhD Thesis Université de Technologie de Compiègne.

9.Meftah, K., Sergent P., Gomi P., 2004. Linear Analysis of a new type of extended Boussinesq model, Coastal Engineering 51, 185-206.

10.Booij, N.,1983. A note on the accuracy of the mild-slope equation. Coastal Engineering, Vol 7, 191-203

11.Dingemans, M.W., 1994. Comparison of Computations with Boussinesq-like models and laboratory measurements. Mast G8-M note, Project 1.

12.Peregrine, D.H.,1967. Long Waves on a beach. Journal of Fluid Mechanics, Vol 27, 815-827.

13.Nadaoka, K., Beji, S., Nakagawa, Y., 1994. A fully dispersive nonlinear wave model and its numerical solutions. Proc. $24^{\text {th }}$ Int. Conf. on Coastal Engineering, 427-441.

14. Massel, S.R., 1993. Extended refraction-diffraction equation for surface waves. Coastal Engineering, Vol 19, 97-126.

15.Madsen, P.A.; Schäffer, H.A., 1998. Higher-order Boussinesq-type equations for surface gravity wave: derivation and analysis. Phil. Trans. R. Soc. Lond. A, vol 356, 31233184.

16.Schäffer, H.A., Madsen, P.A., 1995. Further enhancements of Boussinesq-type equations. Coastal Engineering 26, 1-15. 